\title{
ON THE HARMONIC AND GEOMETRIC MAXIMAL OPERATORS
}

\section{Linden Anne Duffee And Kabe Moen}

Abstract. We examine the harmonic and geometric maximal operators defined for a general basis of open sets in $\mathbb{R}^{n}$. We prove two weight norm inequalities for the harmonic maximal operator assuming testing conditions over characteristic functions of unions of sets from the basis. We also prove a that a bumped two weight $A_{p}$-like condition is sufficient for the two weight boundedness of the harmonic maximal operator.

Mathematics subject classification (2010): 42B25, 42B35, 46E30.

Keywords and phrases: Maximal operators, weighted inequalities.

\section{REFERENCES}

[1] D. CRUZ-URIBE, SFO, The mimimal operator and the geometric maximal operator on $\mathbb{R}^{n}$, Studia Math. 144 (2001) 1-37.

[2] D. CruZ-Uribe, SFO, AND C. J. Neugebauer, The structure of the reverse Hölder classes, Trans. Amer. Math. Soc. 347 (1995) 2941-2960.

[3] D. Cruz-Uribe, SFO, and C. J. Neugebauer, Weighted norm inequalities for the geometric maximal operator, Publ. Mat. 42 (1998) 239-263.

[4] D. Cruz-Uribe, SFO, C. J. Neugebauer, V. Olesen, Norm inequalities for the minimal and maximal operator, and differentiation of the integral, Publ. Mat. 41 (1997) 577-604.

[5] J. DuoandikoetXea, F. J. Martín-Reyes, S. Ombrosi, On the $A_{\infty}$ condition for general bases, Math. Z. 282 (2016), 955-972.

[6] L. Grafakos, L. LiU, C. PÉRez, AND R. H. Torres, The multilinear strong maximal function, J. Geometric Anal. 21 (2011) 118-149.

[7] P. Hagelstein And I. PARISsis, Weighted Solyanik estimates for the Hardy-Littlewood maximal operator and embedding of $A_{\infty}$ into $A_{p}$, J. Geom. Anal. 26 (2016), 924-946.

[8] P. Hagelstein And I. Parissis, Weighted Solyanik estimates for the strong maximal function, preprint http://arxiv.org/abs/1410.3402.

[9] P. Hagelstein, T. LuQue, And I. Parissis, Tauberian conditions, Muckenhoupt weights, and differentiations properties of weighted bases, Trans. Amer. Math. Soc. 367 (2015), 7999-8032.

[10] T. HYTÖNEn AND C. PÉREZ, Sharp weighted bounds involving $A_{\infty}$, Anal. PDE 6 (2012), 777-818.

[11] K. Moen, Sharp weighted bounds without testing or extrapolation, Arch. Math. (Basel) 99 (2012), 457-466.

[12] B. JAWERTH, Weighted inequalities for maximal operators: linearization, localization, and factorization, Amer. J. Math. 108 (1986), 361-414.

[13] L. Liguang And T. LuQue, $A B_{p}$ condition for the strong maximal function, Trans. Amer. Math. Soc. 366 (2014), 5707-5726.

[14] C. PÉREZ, A remark on weighted inequalities for general maximal operators, Proc. Amer. Math. Soc. 119 (1993), 1121-1126.

[15] C. PÉREZ, Weighted norm inequalities for general maximal operators, Publ. Mat. 34 (1990), 1121 1126.

[16] X. SHI, Two inequalities related to geometric mean operators, J. Zhejiang Teacher's 1 (1980) 21-25.

[17] X. YIN AND B. MUCKENHOUPT, Weighted inequalities for the maximal geometric mean operator, Proc. Amer. Math. Soc. 124 (1996), 75-81. 